\title{
Web \& Mobile Application for Particulate Fungi Monitoring
}

\author{
Alcides Alvear Suárez ${ }^{1}$, Juan A. Rivera Morales ${ }^{1}$ \\ ${ }^{1}$ Universidad del Turabo, Puerto Rico, aalvear@suagm.edu, jrivera1054@email.suagm.edu
}

\begin{abstract}
According to the CDC (Center for Disease Control), 22.4\% of Puerto Ricans suffer from asthma and that percentage increases to $25.3 \%$ in children between the ages of 5 and 14 . In the case of allergic asthma, asthma, attacks are commonly caused by pollen and fungal spores in outdoor air. These particles can trigger a reaction in the immune system that controls how the body defends itself. This paper presents the development of an application to show pollen and spores levels collected by certified stations in Puerto Rico. A mobile and web application will be implemented to display the data collected and uploaded to a MySQL database. Also the software currently used to manually count the spores and pollen levels will be redesigned to work with Java and a MySQL database to replace the current one working with VBA and Excel.
\end{abstract}

Keywords—Spores, fungal, mobile, application.

Digital Object Identifier (DOI): http://dx.doi.org/10.18687/LACCEI2015.1.1.168

ISBN: 13 978-0-9822896-8-6

ISSN: $2414-6668$

$1^{\text {th }}$ LACCEI Annual International Conference: “Engineering Education Facing the Grand Challenges, What Are We Doing?” July 29-31, 2015, Santo Domingo, Dominican Republic ISBN: 13 978-0-9822896-8-6

ISSN: 2414-6668

DOI: http://dx.doi.org/10.18687/LACCEI2015.1.1.168 


\title{
Web \& Mobile Application For Particulate Fungi Monitoring
}

\author{
Alcides Alvear Suárez ${ }^{1}$, Juan A. Rivera Morales ${ }^{2}$ \\ ${ }^{1}$ Universidad del Turabo, Puerto Rico, aalvear@suagm.edu \\ ${ }^{2}$ Universidad del Turabo, Puerto Rico, jrivera1054@email.suagm.edu
}

\begin{abstract}
According to the CDC (Center for Disease Control), 22.4\% of Puerto Ricans suffer from asthma and that percentage increases to $25.3 \%$ in children between the ages of 5 and 14 . In the case of allergic asthma, asthma, attacks are commonly caused by pollen and fungal spores in outdoor air. These particles can trigger a reaction in the immune system that controls how the body defends itself. This paper presents the development of an application to show pollen and spores levels collected by certified stations in Puerto Rico. A mobile and web application will be implemented to display the data collected and uploaded to a MySQL database. Also the software currently used to manually count the spores and pollen levels will be redesigned to work with Java and a MySQL database to replace the current one working with VBA and Excel.

Keywords-Spores, fungal, mobile, application.
\end{abstract}

\section{INTRODUCTION}

An application able to show levels of pollen and spores in Puerto Rico is nonexistent. Developing one won't just help individuals with asthma, but anybody mindful of their condition. Those who have been instructed by a specialist, or are currently under medication, can use this application for their own benefit.

Information regarding levels of particulate fungi can be found during the news weather report. Also as a free public service, the National Agency Bureau-American Academy of Allergy Asthma \& Immunology NAB-AAAAI collects all pollen and spores levels from certified stations across the US. Information gathered from San Juan' station can be found at www.aaaai.org/nab [1]. But these services are mostly limited to TV at specific time and PCs.

Pollen and fungus spores are very small and can get inside our house. It is easy to be exposed to outdoor allergens, especially during early hours in the morning. As it is recommended to monitor spores levels, having this mobile application handy can enhance the daily activities, which are limited due the exposure to allergens. The users can manage their conditions better based on the information manually uploaded.

A revamp to the software used to manage the count of each trace of spores will help keep the efficiency in the counting process by having it implemented in Java, which has great portability, speed, standard APIs, etc. A MySQL database will ensure that the data can be accessed by the application. From inputting the traces counts to altering information on stored pollen, spores information and generating reports will be done in the software.

The software's first function is to take a sequence of keystrokes generated by the user, where each represents a spore type, and automatically scan the string for keys and assign the values to a table which are stored in excel.xls format. The second function is to be able to allow access through the program to access these tables and to modify them as needed. Alternatively, it is possible to manually change the values by accessing the tables from the operating system and simply changing them. The third function is to be able to generate charts; this is to be done by storing key values in an access database and then later retrieving these and graphing them with Visual Basic. The fourth and final function is to store images and retrieve them later through the program [2].

The application is relatively new and was started in February 2008, so it is still in its prototype beta version and customized to work with the current system that is in use in Puerto Rico, and it is not a generalized system as it is still in its infancy. But we have seen the need not only in Puerto Rico but in other places as well for a system like this and decided to demonstrate the current version to raise awareness about the software to be developed.

\section{RELATED WORK}

Managing valuable Image-Pro Plus image data and collaborating via the web using the IQbase image database have been reported in [3]. Aerobiology is a branch of biology that studies organic particles, such as bacteria, fungal spores, very small insects and pollen, which are passively transported by the air [4]. One of the main fields of aerobiology has traditionally been to measure and report quantities of airborne pollen as a service to allergy sufferers [4]. A problem has been identified in the process of recording and reporting daily spore counts, as such that is stated as follows: In the day to day management of pollen counts from aerobiological samples of national networks, only a small portion (usually from 12 to $15 \%)$ of the daily microscope slide is read. It is generally believed that, otherwise, too much time will be spent reading slides for a minimal increase in precision [5].

The previous statement is based on an average. And this problem is further worsened by the fact that a study conducted

$13^{\text {th }}$ LACCEI Annual International Conference: "Engineering Education Facing the Grand Challenges, What Are We Doing?" 
states that while the twelve transverse traverse method gave slightly better approximations of the spore concentration, the increase in accuracy may not justify the extra effort required to analyze with this method [6]. These problems give rise to the need for a faster method of reading and storing these counts such as to have more accurate and easier readings.

In Puerto Rico, respiratory conditions constitute an important public health problem by affecting $33.3 \%$ of the children attending school, and from this percentage $51.3 \%$ receive treatment. The East-Central region of the island has been identified by the Puerto Rico Department of Health as one of the areas with the highest prevalence of the disease. This agency reports that the 0 to 17 years old group as highly predisposed. Recognition of the influence of environmental factors in the concentration and dispersal of biological particulates, the use of appropriate air sampler to quantify the density and diversity of the bioallergens in the atmosphere, and recognition of a vulnerable population in the island constitute effective means to design preventive programs, that in the short or long term, will aid in the prevention or control of respiratory conditions caused by the aeroallergens in the air [11].

\section{MATERIALS AND METHODS}

Since this is software the only materials used are software products. These include:

\section{MySQL, Visual Basic 2008, Microsoft Office}

Visual Paradigm Professional Edition 11.2

Burkard: volumetric spore trap (Dynamic)

MK-3: volumetric spore trap (Static)

Nikon 80i Upright Microscope, with fluorescence, DIC, and polarization microscope techniques.

- Camera: 2 mega-pixel colour camera; Effective number of pixels: 1600 x 1200 (1.92 million pixels); Live image: 800 x 600

○ Chip imaging area: $11.8 \mathrm{~mm} \times$ x $8.9 \mathrm{~mm}$; Pixel pitch: 7.4 microns

○ Photo eyepiece magnification: $1 \mathrm{x}$

Davis Instruments 6162 C-WIFI VantagePro2 Plus Cabled WiFi Weather Station

- Measures Outdoor Temperature, Outdoor Humidity, Wind Speed, Wind Direction, Rainfall (Precipitation), Barometric Pressure, Indoor Temperature (console) and Indoor Humidity (console)
Personal Computer: Hewlett Packard HP Pavilion dv2000 AMD Turion64X2 Processor 1.8 GHz clock, 2 Gigabytes of RAM, Windows Vista Business OS 32 bit Version SP1, VIDIA GeForce Go 6150 graphics card. Visual basic 6 was chosen to develop the software since it is the simplest to learn and yet very featured packed and with very few bugs.

\section{STAGES DESCRIPTION}

Figure 1 describes the design presented which is still being developed. It can be partitioned in four stages beginning from gathering the information to finally exhibiting it in a graphic form. The initial two stages are carried out physically and are crucial for the framework to work. After that the redesigned software assumes control to upload the data for the application to fetch the information.

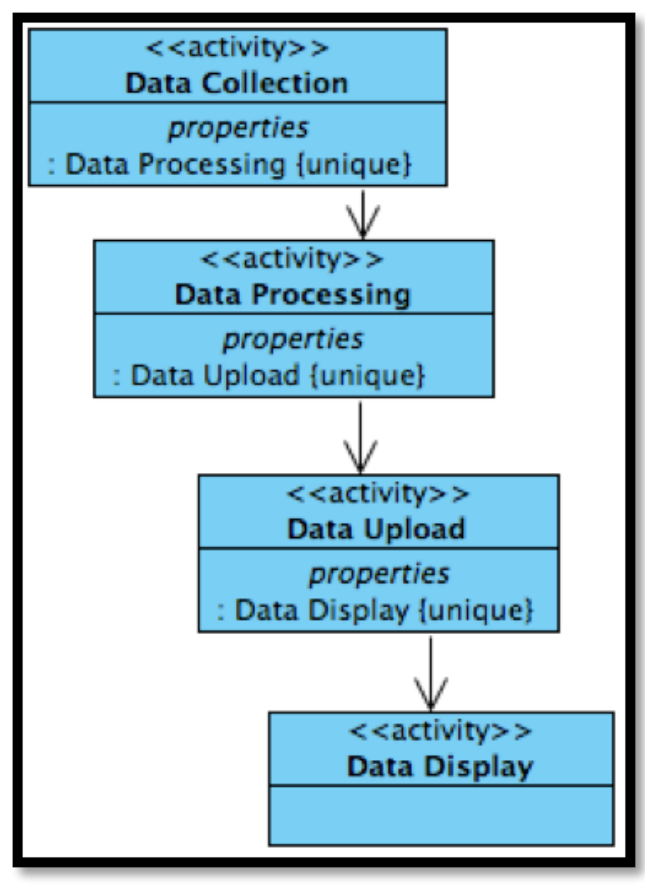

Figure 1. Block Definition Diagram

\section{Data Collection}

Data are collected by a Burkard equipment special collector of air samples. Inside this equipment there is a crystal laminate which is impregnated with silicon where it will remain for 24 hours and impacted with twelve 2-hour impacts; (b) impacted crystal laminate; (c) preparation of the laminate with Calcofluor-white, ready to mount in the microscope; (d) spore viewed through a 100 magnification lens; (e) count process of each spore, that are temporarily registered in a temporary mechanical device called a piano; (f) transfer of the registered data from the piano to the computer in an Excel sheet; (g) spore images are taken according to specific criteria by an expert that is observing through the microscope; up to this point the process is repeated twice; (h) report generation, and lastly results are published in the AAAAI. This process takes approximately 6 hours, and it is susceptible to high error rates 
during the data transfer. A solution to this problem is SporeCount. One of them is installed at the San Juan station in 24-hour mode so the samples are collected daily and processed each morning. To obtain these samples, air is drawn at a rate of 10 liters/min and the particles are caught on a sticky tape.

\section{Data Processing}

This application is a tool that contemplates in its design rules of usability such as the norms of HCI - Human Computer Interaction that make this an easily installable tool and easy to use. SporeCount is a tool created to count organism, whereby organisms can represent anything susceptible to be counted such as: spores in the air, blood cells, people, trees, etc. The test with the SporeCount tool was made with spores of air.

We have studied the HCI literature to identify the characteristics that a software development process should have for it to be considered user-centred. [12], [13], [14], [15], [16] and [17] agree on considering iterative development as a must for a user-centred development process. The complexity of the human side in human computer interaction makes it almost impossible to create a correct design at the first go. Cognitive, sociological, educational, physical and emotional issues may play an important role in any user-system interaction, and an iterative approach is the most sensible way to deal with these issues. Active user involvement and proper understanding of user and task requirements two conditions that can be met by introducing usability techniques that can help software developers with the integration of users into the design process and with the enhancement of requirement activities with specific usability aspects. On the contrary, the first condition (that is, iterative refinement) is an intrinsic characteristic of the software process, and it will be the only requirement for an existing development process to be a candidate for the introduction of usability techniques and activities. When trying to output the activities that constitute a user-centred process, we found that the HCI field offers a heterogeneous landscape of methods and philosophies, like, for instance, usability engineering, usage-centred design, contextual inquiry, and participatory design. Each author attaches importance to a few techniques and the terminology may vary from one author to another. For this reason, we first surveyed the HCI literature to identify the most agreed upon usability activities that should be part of the software development process. We consider the following usability activities [18]:

\section{a. Analysis activities:}

Specification of the context of use

User analysis

Task analysis

b. Design activities

Develop product concept

Prototyping

Interaction design

\section{c. Evaluation activities \\ Usability evaluation}

We preferred to choose techniques that were less alien to software engineering, less costly to apply, and most generally accepted. We have generated a set of potential usability techniques for inclusion in the software development process, where there is at least one technique covering each usability activity and sub-activity that can lead to an improvement in the usability level of the final software product [18].

\section{Data Upload}

A good database design is important for the structure of this application. It is the core of the system since it is where all information is going to be stored, edited and fetched for all the application's features. Fig 2 is a preliminary design of what the relational database of the system is going to be. Advantages:

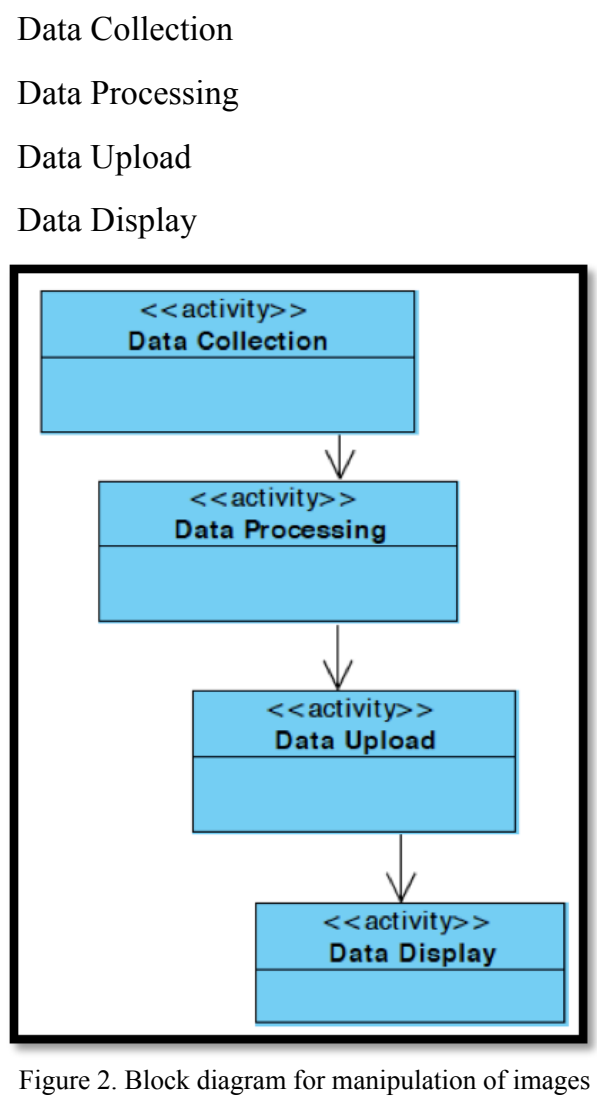

Figure 2 shows with more detail the steps for manipulation of images. It is important to outline here that the database of images is created utilizing the metadata of each image taken; SporeCount permits easy and fast access afterwards, organizing these images automatically.

Each of the possible pollen and spores has an assigned key on the keyboard (currently 58), which are stored in the spores

13 $^{\text {th }}$ LACCEI Annual International Conference: "Engineering Education Facing the Grand Challenges, What Are We Doing?" July 29-31, 2015, Santo Domingo, Dominican Republic 
fungi table of Figure 3. The IDSpore, which refers to the key, is the primary key with latin1_bin collation since they are case sensitive. The data type is varchar since there are both numbers and letters. In the event that the user is not acquainted with these, a popup window with the list of them will be accessible with a click. This list is brought from a table in the database.

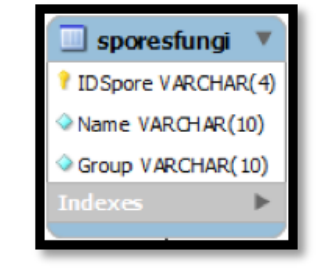

Figure 3. Spores \& Pollen table

After the trace has been processed and the data has been entered on the text area of the input window in Figure 4, the count is taken as a whole string and later separated into individual characters. Then it counts the occurrences of each key, assign that number to the corresponding type of spore and then uploaded. Each trace is uploaded individually to prevent that all traces counted are lost due to a technical malfunction or any unexpected event. The data is stored in a temporary table until all traces have been processed and uploaded. Then they are moved to the official table on which the API will fetch the data for the application. [11].

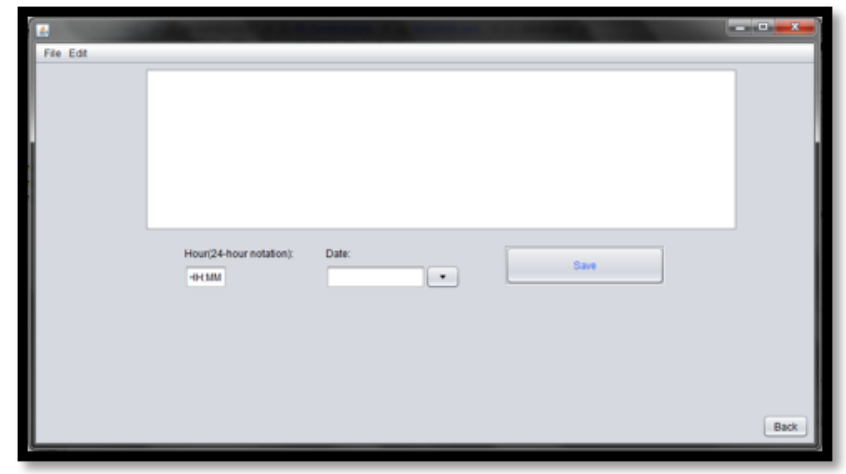

Figure 4. Input Window

To establish the database connection Java Database Connectivity is used (JDBC) [7]. A connection object is created after identifying that the data is valid in addition to the specified date and hour. A statement of the insert query is prepared and later a batch of queries is executed to prevent hitting the database multiple times due to the multiple rows to be inserted. After the batch is executed the program proceeds to check if they were successfully inserted and then the text area of the input interface is cleared for the next trace to count.

\section{Data Display}

Representational State Transfer (REST) is not a standard, but rather an architectural / programming philosophy. It mostly relies, on but is not limited to, the Hypertext Transfer Protocol
(HTTP) and its verbs GET, PUT, POST and DELETE. Its simplicity, fast learning and implementation among other advantages makes it preferable [8]. A REST Application Programming Interface (API) will be implemented to handle the request calls between the application and MySQL. The generated response will be then parsed by the application to display the levels.

\section{DeVElopment Life CyCle}

For the design of this tool a combination of agile methodologies for software development are used: Scrum and Extreme Programming, although without fully using each of these methodologies in the development of this project. One of the development tools used during development was Visual Paradigm Professional Edition 11.2.

Software provides us with many key features such as: receive instant feedback without wasting time manually having to insert certain values to generate a graph in a separate application, minimize human errors (partially because we cannot avoid an error in spore identification among other things) by placing the values automatically in the correct table spaces, minimize time and effort of manually placing values from hardware counters into computer tables among other things. Software is also cost effective and can be easily upgraded to fit certain needs. These features can only effectively be accomplished by the use of computer software. The atmosphere that surrounds the earth is a mixture of essential gases that together form the air. Depending on the weather parameters and the degree of contamination, the air could condense, disperse or transport numerous aerosols, organic particles, virus, bacteria, fungi, pollens, and volatile substances [11].

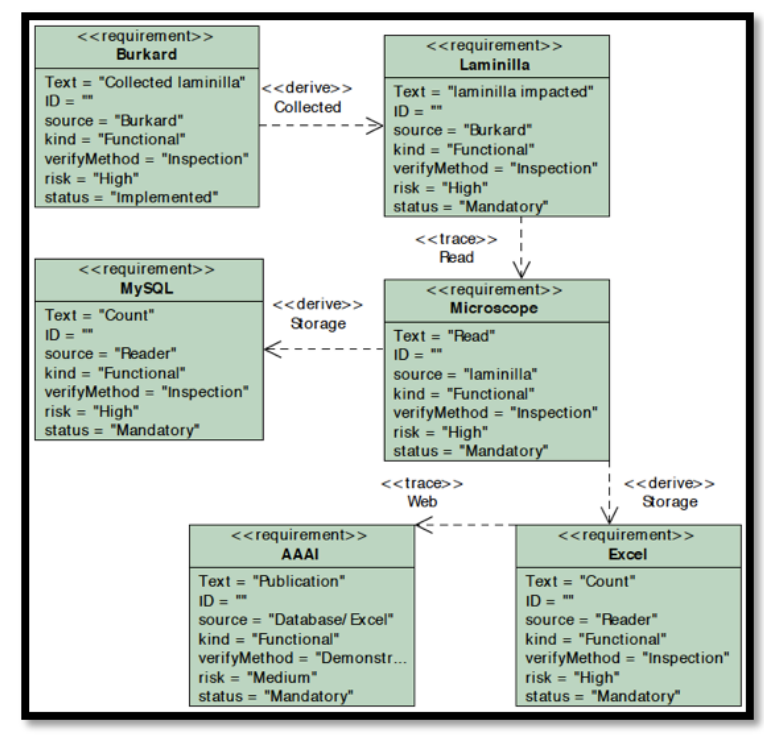

Figure 5. Requirement diagram 
The development cycle used in this project begins with the identification of functional requirements, analysis and depuration thereof. The process is made as indicated by the Extreme Programming methodology. Figures 5 and 6 show, respectively, the functional requirements and the spore reading process from a slide until the data are stored initially on an Excel sheet (current process).

Figure 5 summarizes the functional requirements of the project in six main sections: i) Burkard, is the equipment that hits air every two hours over impregnated silicone; ii) "thinfilm" impregnated with silicone where it has the air samples; iii) microscope, is the equipment used for reading the "thinfilm" before doing the reading; the "thinfilm" are impregnated with calcofluor; iv) MySQL is the database where all the information of daily readings (a slide equals one day of sampling) are stored; v) Excel, is a secondary storage medium of information used to generate other reports in the laboratory; vi) AAAI is the website of the Academy of Asthma Allergy and Immunology; here the daily fungal particulate readings information is published.

This work was a process of reengineering the software used in reading the "thinfilm". This process includes: i) change of programming paradigm, from Visual Basic to Java; ii) use of a database in MySQL, keeping the layout of the data in Excel; and iii) creation of the mobile module to monitor fungal particulate levels in the environment in Puerto Rico.

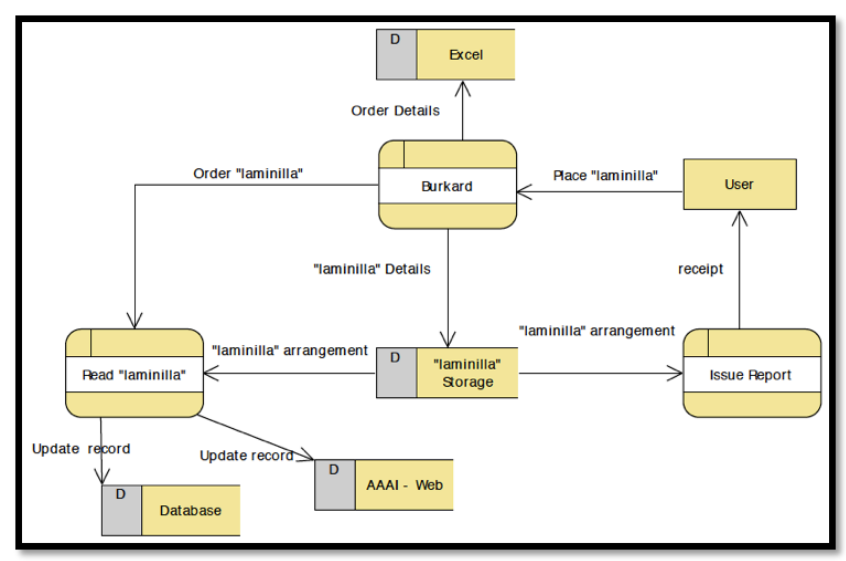

Figure 6. Reading process "thinfilm"

Within the development life cycle and after the first sprint, the database was designed, the Entity Relationship (ER) model is shown in Figure 7.

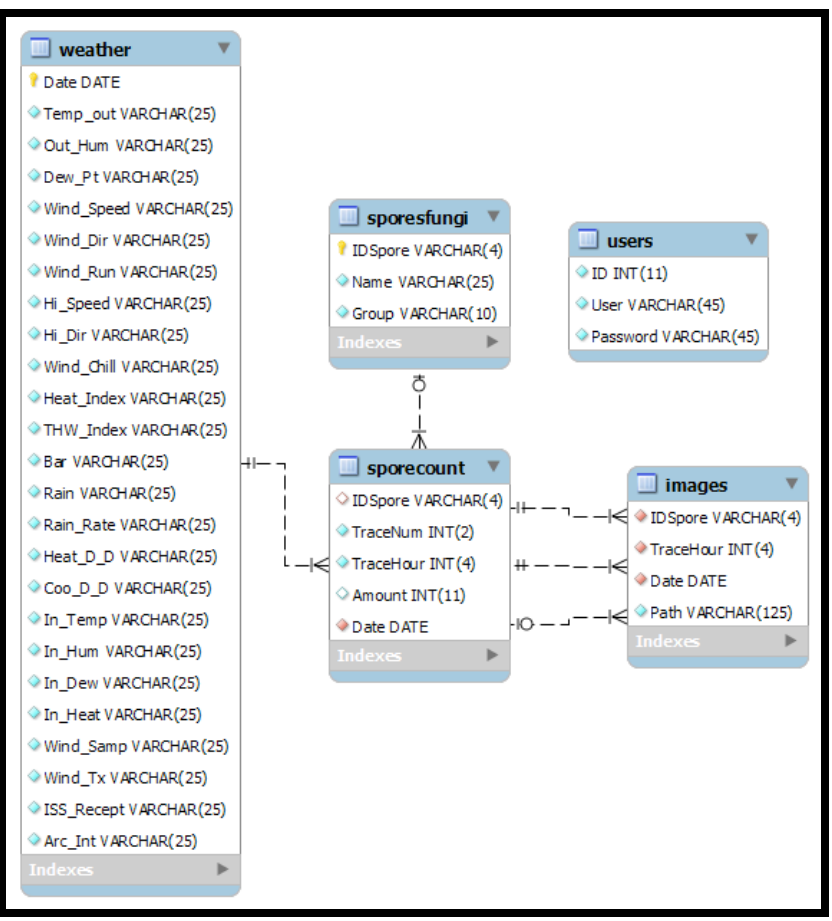

Figure 7. Database ER diagram

The ER model is a relatively simple model involving entities that have information about the factors of time, counting spores, spore types, and possible images that can be obtained from the spores.

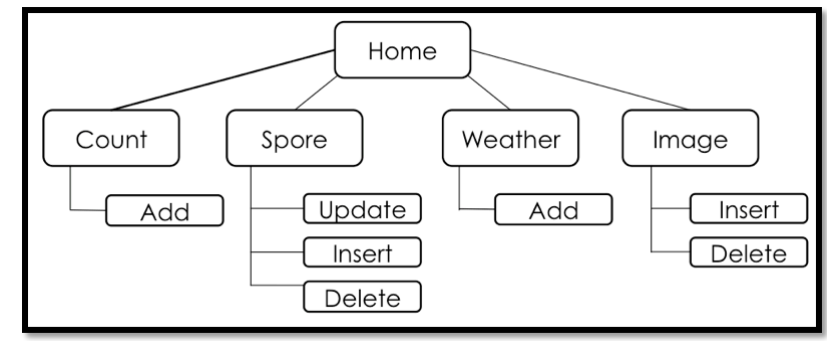

Figure 8. Design of Computer Application

\section{RESULTS}

The versatility of this new application allows fundamental contributions to system administrators and end users know about the fungal particulate on a given day, taking into account other weather conditions such as rain, dew, moisture, temperature and other data, for an eventual decision making about taking a particular allergy treatment or stay home. 


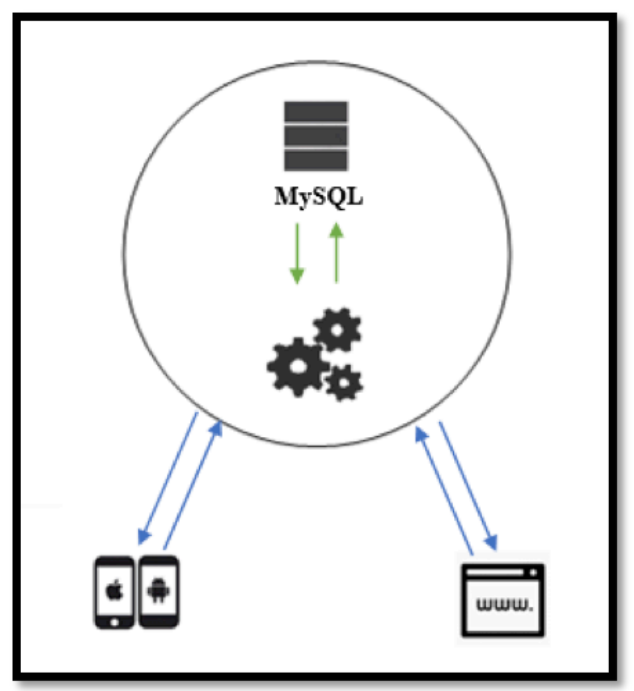

Figure 9. Web \& Mobile to API communication

Figures 9, 10 and 11 show a simple prototype of the web and mobile application. The mobile application will feature three horizontal bars on which the levels are going to be displayed. This is done to more efficiently display data on the reduced display size of a mobile phone. The web page will show three individual gauges for each of the different spore and pollen groups. The phone app will also include a setting option for desired level alert, notifying the user when the levels exceeds their defined setting.

\section{Application}

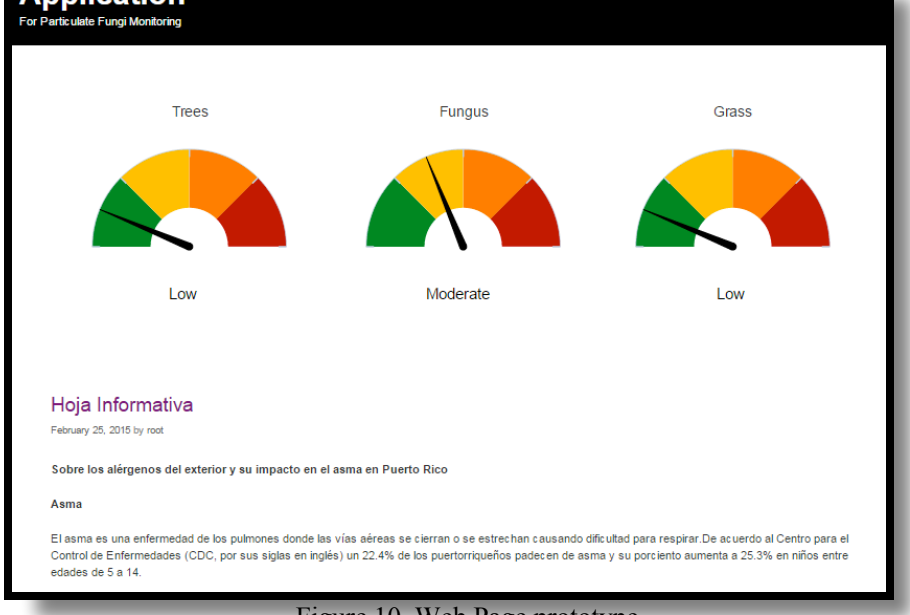

Figure 10. Web Page prototype

Ajax is a design approach and a set of techniques for delivering a highly interactive, desktop-like user experience for Web applications in popular HTML [9] browsers. Ajax, which stands for Asynchronous JavaScript and XML, improves the user's Web application experience while retaining the HTML benefits of server-based application deployment [10].
An Ajax function will handle the calls from the page to retrieve the information on the database. If there is data available it will return an array with the value of each group of spores and pollen. It will then parse the array and assign each value to a variable corresponding to each of the gauges. The variable will be passed as a parameter of the function to draw each gauge automatically when the page loads.

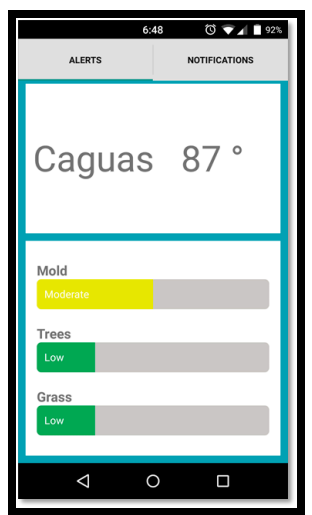

Fig 11. Mobile Application Prototype

To fetch the data on the server an HTTP request is going to be made using the GET method. Similar to the web, the application will receive the JSON object from the API. It will be parsed to retrieve the respective values. The horizontal graphic bars will be drawn using canvas.

\section{CONCLUSIONS}

The software is the optimal solution to facilitate the research process involving spore and pollen counting and monitoring. It will speed up and enhance feedback from this process and allow for more in-depth research to be done in much less time and will improve visual display in mobile devices.

The use of agile development of this software, allowed us to modify and add functional requirements that were appearing in different iterations of the software life cycle product.

In tests conducted under a controlled environment and by end users of this software, it allowed us to make specific changes, without affecting the totality of the whole project.

Although there are no references to publications counting, and reporting of fungal particulate in Latin America and publish their results in the American Academy of Asthma; there are some studies on the analysis of fungal particulate in the air in countries like Colombia, Cuba and Argentina, which could ultimately benefit from software, as we are developing.

The work is innovative and to the authors best knowledge there are not similar studies that can serve as benchmarks. 


\section{ACKNOWLEDGMENT}

Special thanks to Dr. Elizabeth Quintero, and Dr. Benjamin Bolaños for all their contributions and knowledge of spores supplied to this project.

We thank University of Turabo for providing space, equipment and advice whenever we required assistance.

To our friends and all those who in some way contributed their expertise in carrying out this project.

\section{REFERENCES}

[1] www.aaaai.org/nab. Last date consulting: Feb/2/2015.

[2] Media Cybernetics. http://www.mediacy.com/

[3] Comtois P.; Alcazar P.; Néron D. (1999). Pollen counts statistics and its relevance to precision. Aerobiologia,Volume 15, Number 1, 1999, pp. 19-28 (10). Available at: www.ingentaconnect.com/content/klu/aero/1999.

[4] Sterling M.; Rogers C.; Levetin E. An evaluation of two methods used for microscopic analysis of airborne fungal spore concentrations from the Burkard Spore Trap Aerobiologia, Volume 15, Number 1, 1999, pp. 9-18(10). Available at: www.ingentaconnect.com/content/klu/aero/1999.

[5] Larson, K.A. (1993) Prediction of the pollen season with a cumulated activity method. ,Grana, 32,111-114.

[6] Spieksma, F.T. (1991) Aerobiology in the Nineties: Aerobiology and pollinosis, International Aerobiology Newsletter, 34, 1-5.

[7] Deitel, H., \& Deitel, P. (2009). Java ${ }^{\mathrm{TM}}$ How to Program. 8th ed. Prentice Hall.

[8] Guidelines for Implementation of REST. (2011). Enterprise Applications Division of the Systems and Network Analysis Center (SNAC).

[9] Shannon, R. (2012, August 21). HTML Source: HTML Tutorials. Retrieved February 23, 2015, from http://www.yourhtmlsource.com/starthere/whatishtml.html.

[10] Introducing Ajax and OpenAjax. (n.d.). Retrieved February 23, 2015, from http://www.openajax.org/whitepapers/Introducing Ajax and OpenAjax.php.
[11] Alvear A., Quintero E. (2013). Aeroallergens Database Design. International Work-Conference on Bioinformatics and Biomedical Engineering. IWBBIO.

[12] J. Preece, Y. Rogers, H. Sharp, D. Benyon, S. Holland, and T. Carey, Human-Computer Interaction. Addison Wesley, Harlow, England, 1994.

[13] ISO, International Standard ISO 13407. Human-Centred Design Processes for Interactive Systems, ISO, Geneva, Switzerland, 1999.

[14] L. L. Constantine, and L. A. D. Lockwood. Software for Use: A Practical Guide to the Models and Methods of Usage-Centred Design. Addison-Wesley, New York, NY, 1999.

[15] D. Hix, and H.R. Hartson. Developing User Interfaces: Ensuring Usability Through Product and Process. John Wiley and Sons, New York, NY, 1993.

[16]B. Shneiderman. Designing the User Interface: Strategies for Effective Human-Computer Interaction. AddisonWesley, Reading, MA, 1998.

[17] J. Nielsen. Usability Engineering. AP Professional, Boston, MA, 1993.

[18]Ferré X. (2004). Integration of Usability Techniques into the Software Development Process. Universidad Politécnica de Madrid.

13 $^{\text {th }}$ LACCEI Annual International Conference: "Engineering Education Facing the Grand Challenges, What Are We Doing?" 